\title{
Twisted magnetization state at the interface of an antiferromagnetically coupled Fe/Si multilayer as probed by specular and off-specular polarized neutron scattering
}

\author{
Amitesh Paul, ${ }^{1, *}$ Matthias Buchmeier, ${ }^{2, \dagger}$ Daniel E. Bürgler, ${ }^{2}$ Ulrich Rücker, ${ }^{1}$ and Claus M. Schneider ${ }^{2}$ \\ ${ }^{1}$ Institut für Festkörperforschung IFF-4 "Streumethoden," JCNS and JARA-FIT, Forschungszentrum Jülich GmbH, \\ D-52425 Jülich, Germany \\ ${ }^{2}$ Institut für Festkörperforschung IFF-9 “Elektronische Eigenschaften” and JARA-FIT, Forschungszentrum Jülich GmbH, \\ D-52425 Jülich, Germany
}

(Received 15 February 2008; revised manuscript received 16 April 2008; published 12 May 2008)

\begin{abstract}
We have investigated strongly coupled $\mathrm{Fe} / \mathrm{Si}$ multilayers by polarized neutron scattering in specular and off-specular modes. By using the specular reflectivity patterns as a function of the applied field, we have extracted the magnetization angles for layers buried in the stack. In order to explore the magnetization depth profile across the interfaces the neutron data have been combined with micromagnetic simulations. A rigid and/or uniform approximation of the layer magnetization is found to describe the data over a considerable range of the applied field but reveals significant shortcomings around an intermediate field between remanence and saturation. For these intermediate field values, we found (i) close agreement of the magnetization state with a twisted state model, which indicates the formation of exchange-spring-type spin structure; and (ii) depending on the magnetic history of the specimen, an enhanced off-specular magnetic scattering of neutrons at the antiferromagnetic Bragg-peak positions, which indicates the presence of buried domains. However, an extraction of the state of magnetic chirality within a single ferromagnetic layer is in itself challenging and is further complicated as the length scale of these domains becomes smaller than the neutron coherence length.
\end{abstract}

DOI: 10.1103/PhysRevB.77.184409

PACS number(s): 75.70.Cn, 75.60.Jk

\section{INTRODUCTION}

Interlayer exchange coupling ${ }^{1}$ between two ferromagnetic (FM) layers across a nonmagnetic spacer has been one of the remarkable discoveries in thin film magnetism, which together with the giant magnetoresistance ${ }^{2,3}$ laid the foundation for the field of magnetoelectronics, at the same time boosting technological applications in mass storage, nonvolatile memory, and sensors. Microscopically, it is the spindependent reflectivity of the conduction electrons at the interfaces, which gives rise to the formation of spin-dependent quantum well states. This, in turn, causes an oscillatory polarization and a spin-dependent interference effect in the interlayer, resulting in an effective exchange coupling of the two FM layers. Due to its electronic nature, the interlayer exchange coupling strongly depends on the electronic configuration of the interlayer and the interfaces. The quantum interference model predicts an exponential decay of this coupling for insulating spacers, whereas metallic spacers are well known by now to reveal a damped oscillatory coupling. The "intermediate" case, i.e., coupling across a semiconducting layer has been only marginally explored. Yet, the implementation of ferromagnets and semiconductors in devices in an attempt to achieve efficient spin injection makes a combination of these two material classes very interesting. Therefore, the physical mechanisms underlying the interlayer coupling through a semiconductor layer are topics of current scientific relevance.

Within this context, the behavior of the $\mathrm{Fe} / \mathrm{Si}$ system is very peculiar and far from being completely understood. On one hand, epitaxial $\mathrm{Fe} / \mathrm{Si} / \mathrm{Fe}$ trilayers have been found to exhibit a very strong antiferromagnetic (AF) interlayer exchange coupling of $\approx-6 \mathrm{~mJ} / \mathrm{m}^{2},{ }^{4}$ which cannot be explained by the quantum interference model. On the other hand, Si-rich spacers indeed show the predicted exponential decay of the coupling strength with the spacer thickness. ${ }^{4}$ The strong AF interlayer coupling in $\mathrm{Si} / \mathrm{Fe}$ layer systems gives rise to a very interesting magnetization state. ${ }^{5}$ In contrast to a conventional uniform in-plane magnetization often encountered in metallic systems, it was shown by analyzing the frequencies of Brillouin light scattering (BLS) technique that the magnetization is susceptible to assume a twisted state, thereby forming a partial domain wall parallel to the $\mathrm{Fe} / \mathrm{Si}$ interfaces. Such a state is realized when there is a competition between the torques exerted in the bulk by the external applied field favoring parallel alignment and at the interfaces by the strong AF coupling. The total energy in the twisted state configuration is lower than that for a uniform magnetization state because the gain in interlayer exchange energy prevails the increase in the intralayer exchange energy.

The first evidence for the formation of a twisted magnetization state was observed in ferrimagnetic $\mathrm{Gd} / \mathrm{Fe}$ multilayers (MLs) by Mössbauer spectroscopy. ${ }^{6}$ The system minimizes the Zeeman energy by progressively rotating the $\mathrm{Fe}$ moments from the interface to the center of the layer toward the applied field direction at a critical field value. In another experiment, the surface nucleation of a twisted magnetization state was demonstrated, which was again in a ferrimagnetic $\mathrm{Gd} / \mathrm{Fe}$ multilayer. Simultaneous measurements of surface- and bulk-sensitive element-specific hysteresis loops by magnetic circular dichroism showed that the twist can indeed propagate throughout the bulk part of the layers. ${ }^{7}$ The existence of the twisted state throughout the volume of a ferromagnet-nonmagnet ML system, such as Fe/Si MLs, has not yet been demonstrated. A previous work based on BLS measurements ${ }^{5}$ was limited to $\mathrm{Fe} / \mathrm{Si} / \mathrm{Fe}$ trilayers due to the limited information depth of BLS of about $100 \mathrm{~nm}$. If the magnetization profile in an entire ML structure is to be studied, a depth-sensitive technique is more appropriate in order to address the buried layers. 
Polarized neutron reflectivity (PNR) is a depth-sensitive technique and furthermore also possesses vector magnetometric capabilities. ${ }^{8}$ Therefore, a twisted ground state configuration in a multilayer-extending from the interfaces through the FM layer thickness - can be conveniently analyzed by this approach. PNR data on $\mathrm{Gd} / \mathrm{Fe}$ MLs only showed an increase in the magnetization component $M_{\perp}$ perpendicular to the applied field direction, signifying a coherent rotation of the $\mathrm{Fe}$ moments around the compensation temperature. ${ }^{9}$ Magnetic spirals in buried layers of exchangespring-type systems have been observed earlier by PNR for a combination of hard and soft magnetic layers. ${ }^{10,11}$ However, the investigation of such complex noncollinear spin structures by any depth-sensitive technique has thus far not been extended to the case of a ferromagnet-semiconductor, i.e., ferromagnet-nonmagnet, combination. These systems are particularly interesting as they do not exhibit a compensation temperature, around which the twisting is observable.

The motivation of this investigation is to examine the twisted state with a complementary method, i.e., by probing the Fourier transform of magnetization profile with PNR rather than analyzing via BLS frequencies the torques acting on the magnetization at the interfaces and in the bulk. ${ }^{5}$ Moreover, we want to show the existence of the twisted state in all FM layers throughout a ML. Thus, in the present paper, we investigate the magnetization state of a strongly coupled epitaxial $\mathrm{Fe} / \mathrm{Si}$ ML by PNR in the specular and off-specular scattering modes. While the averaged magnetization angles are found to agree with a model by assuming rigid and/or uniform approximation over a large field range, significant discrepancies are found around intermediate field values. In this intermediate field range between remanence and saturation, we find a much better description of the scattered signal with the twisted state model, which is also obtained in micromagnetic simulations after energy minimization of different magnetic states. Superconducting quantum interference device (SQUID) data also agree with these simulations. However, extracting a refined layer magnetization profile still remains a challenging task. The situation is even more complicated by the presence of domain states, which essentially also depend on the field history of the specimen.

\section{EXPERIMENTAL DETAILS}

\section{A. Sample preparation}

Multilayers with the full layer sequence $[\mathrm{Fe}(28.0 \mathrm{~nm}) / \mathrm{Si}(1.3 \mathrm{~nm})]_{6} / \mathrm{Fe}(28.0 \mathrm{~nm})$ were prepared by thermal e-beam evaporation and epitaxial growth onto $\mathrm{GaAs}(001)$ substrates with a buffer layer of $\mathrm{Fe}(1.0 \mathrm{~nm}) / \mathrm{Ag}$ $(150 \mathrm{~nm})$. The details of the sample preparation were previously given. ${ }^{5}$ Alloying of $\mathrm{Si}$ with Fe was suppressed by depositing the Si spacers at room temperature, yet maintaining the epitaxial nature throughout the entire ML stack. An antireflection coating of $\mathrm{ZnS}(4.2 \mathrm{~nm})$ formed a cap layer to prevent the stack from oxidation.

\section{B. Neutron scattering}

The neutron scattering geometry is shown in Fig. 1. In the

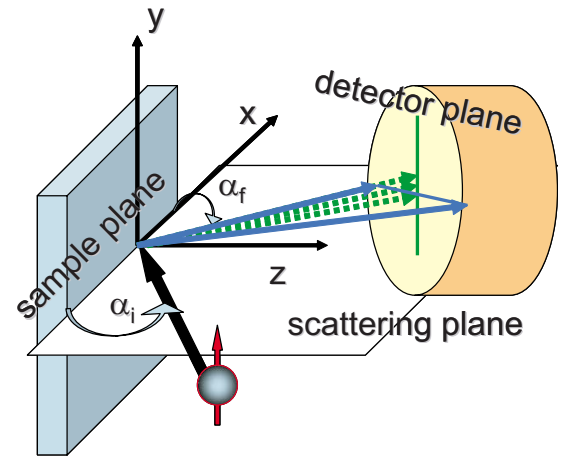

FIG. 1. (Color online) Schematic of the neutron scattering geometry. The beam is collimated in the reflection plane and relaxed along the $y$ axis.

specular scattering geometry (i.e., angle of incidence $\alpha_{i}$ equal to the exit angle $\alpha_{f}$ ), the reflectivities follow from energy and in-plane momentum conservation laws as normal wave vector transfers $Q_{\perp}=2 \pi^{\prime} \lambda\left[\sin \left(\alpha_{i}\right)+\sin \left(\alpha_{f}\right)\right]$ are probed. We consider a sample surface in the $x-y$ plane and the $z$ axis along the surface normal. Off-specular scattering contributions along the in-plane momentum transfer vector $Q_{\|}$ $=2 \pi^{\prime} \lambda\left[\cos \left(\alpha_{f}\right)-\cos \left(\alpha_{i}\right)\right]$ arise; however, when the in-plane translational symmetry is broken by interface waviness (roughness) or by magnetic domains on a length scale shorter than the in-plane projection of the neutron coherence length $l_{\|}$(Refs. 12 and 13) along $Q_{\|}\left(=Q_{x}, Q_{y}\right)$.

The resolutions in the in-plane components of the momentum transfer vector $\left(Q_{\|}\right)$can be expressed as $\boldsymbol{\varsigma}_{x, y}$ $=2 \pi / \delta Q_{x, y}$. The in-plane projection (parallel projection) of neutron coherence $l_{\|}$is related to the respective resolutions $\left(\sim \boldsymbol{\varsigma}_{x} \cdot \boldsymbol{\varsigma}_{x}\right)$ along the $x$ and $y$ directions. In the present experimental geometry, only $Q_{x}$ is resolved, whereas the signal along $Q_{y}$ is integrated (collimation along the $y$ axis is relaxed). The typical spread of $s_{x}$ varies from a few nanometers to a few micrometers. On the other hand, $l_{\perp}$ (perpendicular projection), which is also related to $\varsigma_{z}\left(=2 \pi / \delta Q_{z}\right)$ (due to the possibility of employing a higher angular resolution), has a relatively much higher extension (in several microns) and thus the scattering can be considered coherent along the thickness of the specimen.

\section{Specular scattering}

In the following, we define the longitudinal magnetization component $M_{\|}$as being parallel to the applied field direction $\left(M_{\|}=\vec{M} \cos \phi_{A}\right)$ and the transversal magnetization component $M_{\perp}$ as perpendicular to the applied field direction $\left(M_{\perp}=\vec{M} \sin \phi_{A}\right)$, where $\phi_{A}$ is the angle between the magnetization direction $\vec{M}$ and the applied field $\vec{H}_{a}$.

Due to the interaction between polarized neutrons and the magnetic moments in the specimen, PNR is sensitive to the in-plane magnetization for a homogeneous film. Neutron scattering measures the average of the in-plane magnetization vector, which is essentially the incoherent average over several coherent volumes (each $\approx 5-10 \mu \mathrm{m}$ in diameter, as defined by $l_{\|}$). The projection of the neutron coherence length along the sample plane is usually smaller than the illumi- 
nated sample surface. The averaging of the local magnetization on a length scale smaller than $l_{\|}$is coherently summed, whereas averaging over the total illuminated area is incoherently summed. The signal along $Q_{y}$ is integrated and thereby results in incoherent average along the $y$ direction.

The neutron interaction potential is given by

$$
V=\frac{2 \pi \hbar^{2}}{m}\left(\rho_{n} \pm \rho_{m}\right)=\frac{2 \pi \hbar^{2}}{m} N b_{n} \pm \vec{\sigma} \cdot \vec{B},
$$

where $N$ is the atomic density, $b_{n}$ is the coherent nuclear scattering length, $m$ is the neutron mass, $\vec{B}$ is the internal magnetic field of the specimen, and $\vec{\sigma}$ represents the operator associated with the Pauli spin matrices. The scattering-length densities (SLD) of a magnetic specimen are given by either the sum or difference of the nuclear $\left(\rho_{n}\right)$ and magnetic $\left(\rho_{m}\right)$ components. The \pm signs refer to the spin-up and spin-down states of the incident neutron with respect to the polarization of the specimen.

The reflectivity is calculated by applying the timeindependent Schrödinger equation for the neutron wave function inside and outside the specimen. One can solve the equation by taking into account the spin dependences of the scattering potential and the neutron wave function. In this way, we get the expressions for non-spin-flip (NSF) and spin-flip (SF) scatterings. The NSF scattering amplitude provides information about $\rho_{n} \pm \rho_{m} \cos \phi_{A}$, i.e., the magnetization component $M_{\|}$parallel or antiparallel to the applied field. The SF channels measure $\rho_{m}^{2} \sin ^{2} \phi_{A}$, i.e., the magnetization component $M_{\perp}$ perpendicular to the applied field, if the domain size is larger than $l_{\|}$. In this approach, the magnetic scattering length $b_{m}\left(\rho_{m}=N b_{m}\right)$ includes the magnetization $M$ and the neutron magnetic moment $\mu_{N}$. The magnetization component parallel to the scattering vector (i.e., normal to the sample surface) is not visible to neutrons as Maxwell's equation $(\nabla \cdot \vec{B})$ requires the out-of-plane component of $\vec{B}$ across the interface to be continuous. The variation in the magnetization vector (in-plane amplitude and direction) with depth can be extracted within the dynamical format (the phase information, however, remains implicit) by using a conventional reflectivity fitting analysis, as the reflection amplitude is related to the Fourier transform of the SLD depth profile. Therefore, the specular reflectivities in the NSF channels $\left(R_{++}\right.$and $\left.R_{--}\right)$are due to periodicities of the structure and magnetization components collinear to $H_{a}$, whereas reflectivities in the SF channels $\left(R_{+-}\right.$and $\left.R_{-+}\right)$are exclusively of magnetic origin and correspond to in-plane magnetization components perpendicular to $H_{a}$. A magnetization reversal via rotation is identified by an increase in the specular reflectivities in the SF channels, which map the inplane magnetization components perpendicular to the guiding field $H_{a}$. On the other hand, a reversal by domain nucleation and propagation does not lead to enhanced SF reflectivities (specular), as long as the domain sizes are smaller than $l_{\|}$, but can produce off-specular signal in the SF channels.

\section{Off-specular scattering}

As long as the condition of translational invariance holds (within a certain coherent volume), it will not lead to any off-specular scattering. Thus, the scattered intensity that one measures, in the case of specular scattering, is the depth variation of laterally average interaction potential from different coherent volumes (defined by $Q_{\|}=0$ ). However, when the invariance is broken (but on a length scale smaller than $\left.l_{\|}\right)$, off-specular scattering may be seen, which measures the fluctuations around the mean value of the laterally averaged interaction potential.

Due to the influence of dynamical effects (multiple scattering due to refraction and total external reflection) close to the critical angle of total reflection, the distorted wave born approximation (DWBA) is used instead of the Born approximation. In this approach, the potential operator in each layer can be decomposed into a sum of the reference potential and perturbation contribution in the form

$$
\overline{V(x, y, z)}=\overline{\langle V\rangle(z)}+\overline{V_{\text {perturbed }}(x, y, z)},
$$

where $\overline{\langle V\rangle(z)}$ denotes the reference potential averaged over all lateral coordinates, which is responsible for specular scattering, while $\overline{V_{\text {perturbed }}(x, y, z)}$ causes the off-specular scattering signatures. The perturbed potential includes the nuclear and magnetic scattering length density fluctuations. Offspecular scattering affected by dynamical effects (close to the critical angle of total reflection) can be taken into account within the reflection and transmission coefficients involved. Furthermore, involving linear combinations of the Pauli spin matrices and unit matrices, the lateral Fourier transform of the perturbed potential can be written as the Fourier transform of the pair correlation functions involving nuclear and magnetic fluctuations.

In our model the mean magnetization with respect to the applied field varies from region to region around a mean angle $\phi_{A}$ with a Gaussian distribution of width $\Delta \phi_{A}$. In this model, both the components of magnetization parallel (along the $y$ axis) and perpendicular (along the $x$ axis) to $H_{a}$ will contribute to the diffuse scattering signal. ${ }^{12}$

Following summation over the coherence regime in $Q_{x}$ and integration in $Q_{y}$, the Fourier transform of the pair correlation functions can be written as Lorentzian functions for $Q_{x}$, giving the amplitude of fluctuation of magnetization $\| / \perp$ to the field direction. With an increase in the amplitude of fluctuations, the perpendicular components increase faster than their parallel counterparts (greater intensities in the SF channels), which enables the unique determination of $\Delta \phi_{A}$ (by using proper recalibration factors). The simulations of the intensity maps within the DWBA is essentially a sum of the specular scattering as well as the off-specular scattering from structural correlations and magnetic fluctuations along and perpendicular to the applied filed. However, we show the maps corresponding to the SF channels only, where the magnetic scattering is exclusive.

\section{Instrument}

The neutron scattering experiments were performed at the polarized neutron reflectometer with polarization analysis 


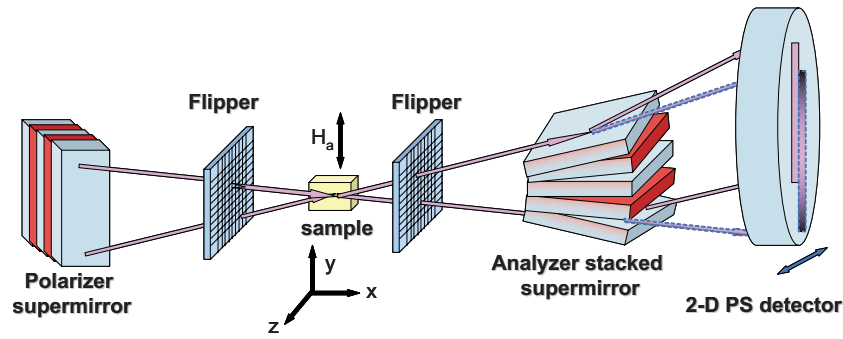

FIG. 2. (Color online) Schematics of the experimental setup at the HADAS neutron reflectometer. The perpendicular alignment of the analyzer with respect to the polarizer does not affect the horizontal component of neutron beam but offers a large angular acceptance, which is relevant for diffuse scattering measurements.

HADAS at the Jülich research reactor FRJ-2 (DIDO). For a wavelength of $4.52 \AA$, a $Q$ resolution $\Delta Q / Q \approx 1.0 \%$ was chosen, which is typical for such reflectivity measurements. Figure 2 depicts the schematic of the HADAS reflectometer. Collimation is provided by two slits in front of the sample and two Mezei spin flippers were used to reverse the neutron spin projection on the quantization axis. The stack of analyzers is turned by $90^{\circ}$ with respect to the polarizer supermirror in order to get a larger angle of acceptance for the scattered beam, which is relevant for diffuse scattering. This geometry does not affect the horizontal component of the beam but results in a vertical shift on the detector, owing to the reflecting supermirrors.

We present the specular data (or reflectivities) as along $Q_{z}$ for a constant $Q_{x}$ but prefer to present our off-specular data as intensity maps $\left(\alpha_{i}\right.$ and $\left.\alpha_{f}\right)$ instead of $Q_{x}$ scans at an almost constant $Q_{z}$ value (rocking scans) because the amplitudes of reflection and transmission coefficients (which determines the intensity) are themselves angle dependent. Conventional perpendicular cuts through Bragg sheets (at constant $Q_{z}$ ) may not include intensities toward higher $Q_{\|}$values. The situation is more complicated in the case of vertically uncorrelated structures, ${ }^{14}$ wherein an enhancement of the off-specular scattering is seen only near the critical angle and suffers significant losses away from the critical angle. Thus, we follow the more general approach in presenting the data and its simulation.

The resolution of the wavelength, the divergence of the beam, and the illumination effects on the size of the sample were all convoluted with the simulation. For our instrument, $l_{\|}$exceeds $20 \mu \mathrm{m}$, but the resolution of the two-dimensional position-sensitive detector defines an upper limit of about $5-10 \mu \mathrm{m}$ for the resolvable lateral structure size, whereas sizes that are even $<1 \mu \mathrm{m}$ can be estimated as long as the scattered intensity is sufficient enough.

\section{EXPERIMENTAL RESULTS}

\section{A. Magneto-optic Kerr effect and superconducting quantum interference}

The remagnetization behavior was investigated by means of a commercial SQUID and a magneto-optic Kerr effect (MOKE) setup, as described in Ref. 15. The MOKE signal

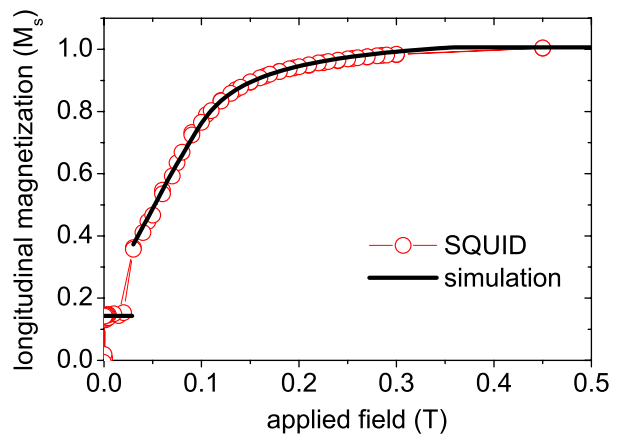

FIG. 3. (Color online) SQUID hysteresis loop of a Fe/Si ML measured in the easy axis configuration (red connected symbols) compared to the fitted curve (black line).

roughly corresponds to an averaged magnetization direction within the whole area illuminated by the laser spot (large coherence length); yet, it is limited to an information depth on the order of $30 \mathrm{~nm}$. On the other hand, SQUID typically measures the bulk net magnetization of the complete sample along the external field direction. The complementary information derived from both magnetization measurements, as been used in conjunction as input for micromagnetic simulations in order to extract various energy parameters.

A phenomenological description of the magnetization curves yields the coupling energy per unit area as

$$
E=-J_{1} \cos (\theta)-J_{2} \cos ^{2}(\theta),
$$

where $J_{1}$ and $J_{2}$ are the bilinear and biquadratic coupling constants, respectively, and $\theta$ is the angle between the two layer magnetizations on both sides of the spacer.

Figure 3 shows half of the SQUID remagnetization loop in the field range of $0<H_{a}<0.5 \mathrm{~T}$. The data have been recorded with $\vec{H}_{a}$ along an easy axis of the magnetocrystalline anisotropy, i.e., parallel to $\mathrm{Fe}[001]$. The diamagnetic contribution stemming from the substrate and causing a linear background has been subtracted based on the slope of the curve in the field range of $1-3 \mathrm{~T}$. At low fields $(0-0.020 \mathrm{~T})$, we observe an antiparallel alignment of the magnetizations of neighboring layers, and the total net magnetization of the ML is collinear with the external field direction. This situation is indicative of a predominantly AF coupling $\left(\left|J_{1}\right|\right.$ $\left.>\left|2 J_{2}\right|\right)$ and is typically encountered in ML structures, which contain an odd number of FM layers. A spin-flop transition manifesting itself as a discontinuous change in the magnetization into a canted configuration takes place at about $0.02 \mathrm{~T}$. Consecutively, the magnetization continuously turns into the field direction and slowly saturates above $0.3 \mathrm{~T}$. The fact that magnetic saturation is not reached at a clearly defined field value, but instead the slope of the curve still very slowly changes at high fields-i.e., in terms of the Ehrenfest classification scheme, the saturation is not a second but a third order phase transition-is a typical feature of the twisted magnetization state.

We have fitted the remagnetization loops by taking the twisted state model into account by using the freely available OOMMFF (Ref. 16) micromagnetic code. We assumed literature values for the intralayer exchange $A=1.98 \times 10^{-11} \mathrm{~J} / \mathrm{m}$, 

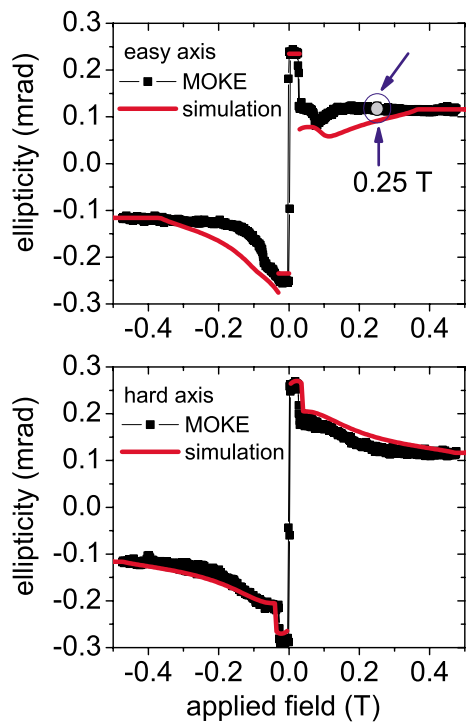

FIG. 4. (Color online) Full MOKE hysteresis loops of a Fe/Si ML measured with the external field applied along the easy axis and hard axes of the Fe layers, respectively (red lines). The simulated curves are shown as black lines. The black dot in the easy axis curve indicates the critical field, at which the neutron measurements were carried out after negative (down arrow) and after positive (up arrow) saturation.

a saturation magnetization of $M_{s}=1.7 \times 10^{6} \mathrm{~A} / \mathrm{m}$, and a magnetocrystalline anisotropy of $K_{1}=45 \mathrm{~kJ} / \mathrm{m}^{3}$, while treating the interlayer coupling parameters $J_{1}$ and $J_{2}$, which we assumed to be identical for all of the six interlayers, as adjustable parameters. As the lateral sample dimensions are very large compared to the layer thicknesses, the stray field energy has been described by an effective uniaxial shape anisotropy of the strength $K_{u}=1 / 2 \mu_{0} M_{\text {eff }}^{2}{ }^{15}$ We find coupling values of $J_{1}=-2.3 \mathrm{~mJ} / \mathrm{m}^{2}$ and $J_{2}=-0.3 \mathrm{~mJ} / \mathrm{m}^{2}$ with confidentiality errors of less than $0.1 \mathrm{~mJ} / \mathrm{m}^{2}$. By taking into account that as a result of the changing layer quality through the stack and an uncertainty of the Si layer thicknesses of about $0.5 \AA$, the coupling values are expected to vary from interlayer to interlayer; the excellent agreement between the fitted and experimental curves is surprising.

MOKE remagnetization loops provide additional information as the method's information depth is limited to the two topmost Fe layers, while SQUID determines the average magnetization of all of the layers. MOKE loops with the field applied parallel to easy and hard axes are presented in Fig. 4. The MOKE ellipticity obtained from the longitudinal geometry $\left(\epsilon_{\text {long }}\right)$ has been modeled from the calculated magnetization angles by taking into account the first order terms linear in $\cos \phi_{A}$ and second order quadratic terms proportional to $\cos \phi_{A}^{*} \sin \phi_{A}$, i.e.,

$$
\epsilon_{\mathrm{long}}=\sum_{i}\left(l_{i} \cos \phi_{A, i}+q_{i} \cos \phi_{A, i} \sin \phi_{A, i}\right) .
$$

Here, $l_{i}$ and $q_{i}$ are phenomenologically the first and second order MOKE coefficients, which have been extracted from the easy axis loops, and the layer index $i$ is described by the Fe layer. ${ }^{17}$
The easy axis MOKE loop looks similar to the SQUID loop presented in Fig. 3 with the notable difference that the measured Kerr ellipticity is larger for the antiparallel state than for the saturated state. This is due to the opposite signs of the MOKE contributions generated in the topmost and the adjacent magnetic layer. Moreover, there is a distinct asymmetry between the positive and negative applied field directions, which is of magneto-optical origin due to significant quadratic contributions to the signal. ${ }^{15}$ For comparison, we also plot the calculated MOKE curves (black lines), for which we employed the magnetic parameters listed above, optical and magneto-optical material constants for Fe from Refs. 18 and 19, and an index of refraction $n_{Z n S}=2.342$ $+3.645 i$ for the $\mathrm{ZnS}$ cap layer. ${ }^{19}$ We neglected the thin $\mathrm{Si}$ spacer layers. The calculated Kerr ellipticity has been scaled by a factor of 1.15 in order to match the saturation Kerr angle of the experimental data. Obviously, the MOKE loop shows a saturation field of about $0.2 \mathrm{~T}$, which is smaller than that of the SQUID data $(>0.3 \mathrm{~T})$ and the calculation based on coupling constants $J_{1}$ and $J_{2}$ derived from fitting the SQUID data. Therefore, the coupling mediated by the topmost $\mathrm{Si}$ spacer is weaker than the average coupling strength of the complete ML. However, it should be noted that this does not mean a large variation in the coupling strengths. For the twisted state, small variations in the coupling strength lead to large changes in the saturation field; e.g., a $25 \%$ stronger coupling corresponds to about a doubling of the saturation field. ${ }^{5}$

\section{B. Neutron scattering}

\section{Neutron specular scattering}

In order to obtain a more detailed picture of the magnetization reversal process, we performed a series of PNR measurements on one side of the hysteresis loop at six different field values with the field being applied along an easy axis. Prior to the field sweeps, the specimen was saturated at -0.4 T. The specular intensity in Fig. 5 along the line $\alpha_{i}$ $=\alpha_{f}$ shows the first six orders of nuclear Bragg peaks corresponding to the bilayer thickness [best visible for saturating fields in Figs. 5(e) and 5(f)]. The half-order AF peaks are visible in Fig. 5(a) at $H_{a}=0.01 \mathrm{~T}$ close to the remanent state.

\section{Neutron off-specular scattering}

Next, we turn to the off-specular scattering data. Figure 6 shows the SF intensity $\left(R_{-+}\right)$maps as a function of $\alpha_{i}$ and $\alpha_{f}$ at different representative fields $H_{a}$. An enhanced offspecular intensity in the SF channel gradually appears in Fig. $6(\mathrm{~d})$ at $H_{a}=0.25 \mathrm{~T}$ close to angles $\alpha_{f} \approx 15-25 \mathrm{mrad}$. These enhancements are mainly located at the respective AF halforder Bragg peak positions as Bragg sheets, which are distinctly visible in comparison to the intensities in the saturated state [Fig. 6(f)]. An asymmetry in the intensities of these diffuse Bragg sheets due to different critical angles of total reflection for incident (spin-up and/or spin-down) and exit (spin-down and/or spin-up) neutrons with respect to the magnetization direction of Fe layers is also visible. The sizable 


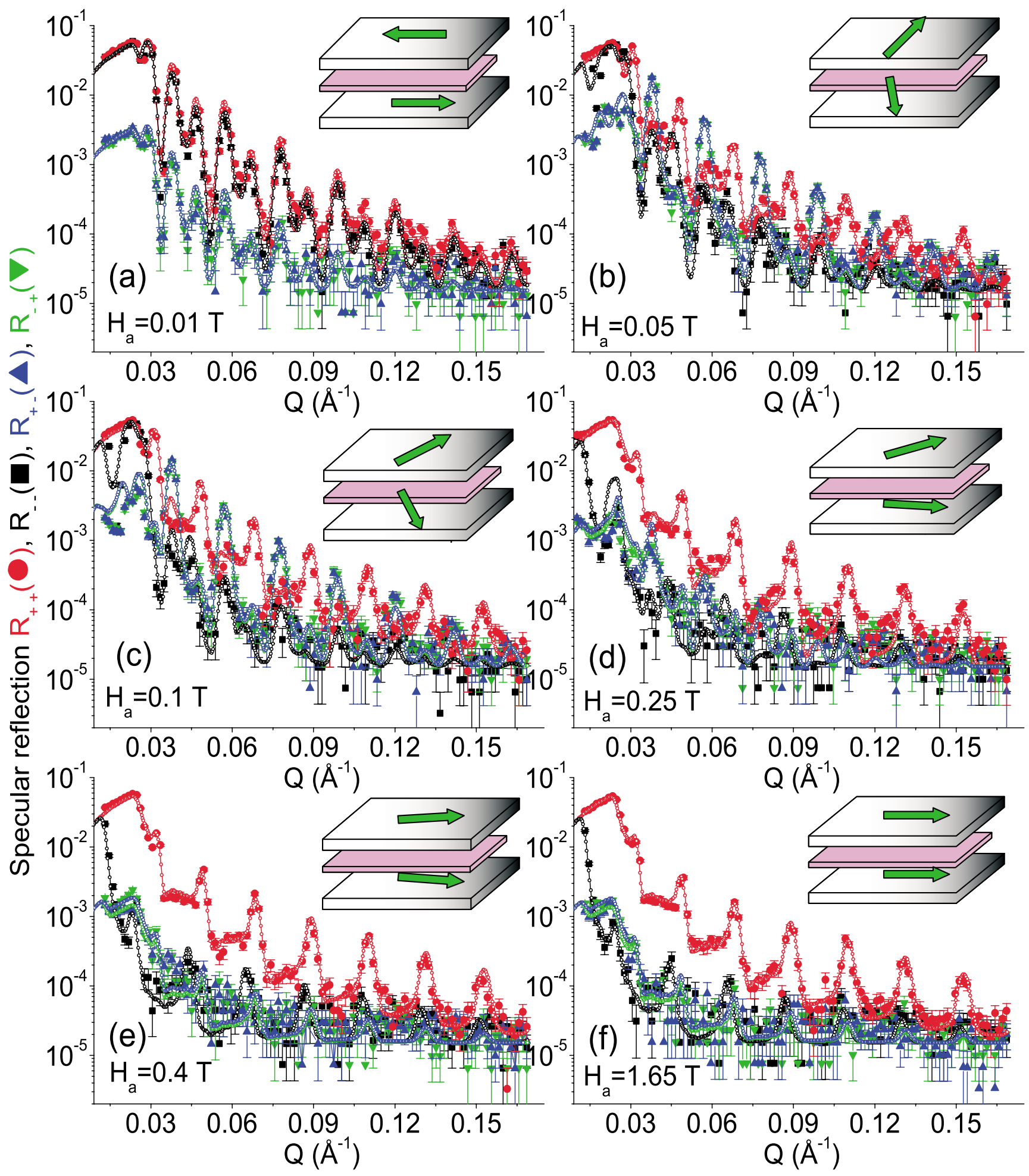

FIG. 5. (Color online) Specular reflectivity patterns for the NSF $\left[R_{++}\right.$(red) and $R_{--}$(black) $]$and SF $\left[R_{-+}\right.$(green) and $R_{+-}$(blue) $]$channels measured for a Fe/Si ML at different $H_{a}$ as indicated after negative saturation. The solid symbols are data and the open circles are fits to a rigid-state model. The insets show the magnetization alignment of the two adjacent Fe layers in the ML stack.

off-specular SF intensity confirms that $M_{\perp}$ is indeed laterally inhomogeneous; i.e., it exhibits a laterally random variation in the magnetization directions around their mean value from domain to domain on a length scale smaller than $l_{\|}$of the neutron beam. Moreover, we note that the diffuse scattering intensities are observed in the Bragg sheets, indicating only that the in-plane variations are coherent throughout the ML stack. At saturation, the intensity resulting from diffuse scattering events disappears, which confirms its purely magnetic origin. The lateral correlation length for such a variation can be estimated from the simulation of the SF intensity maps under the DWBA. ${ }^{12}$ 

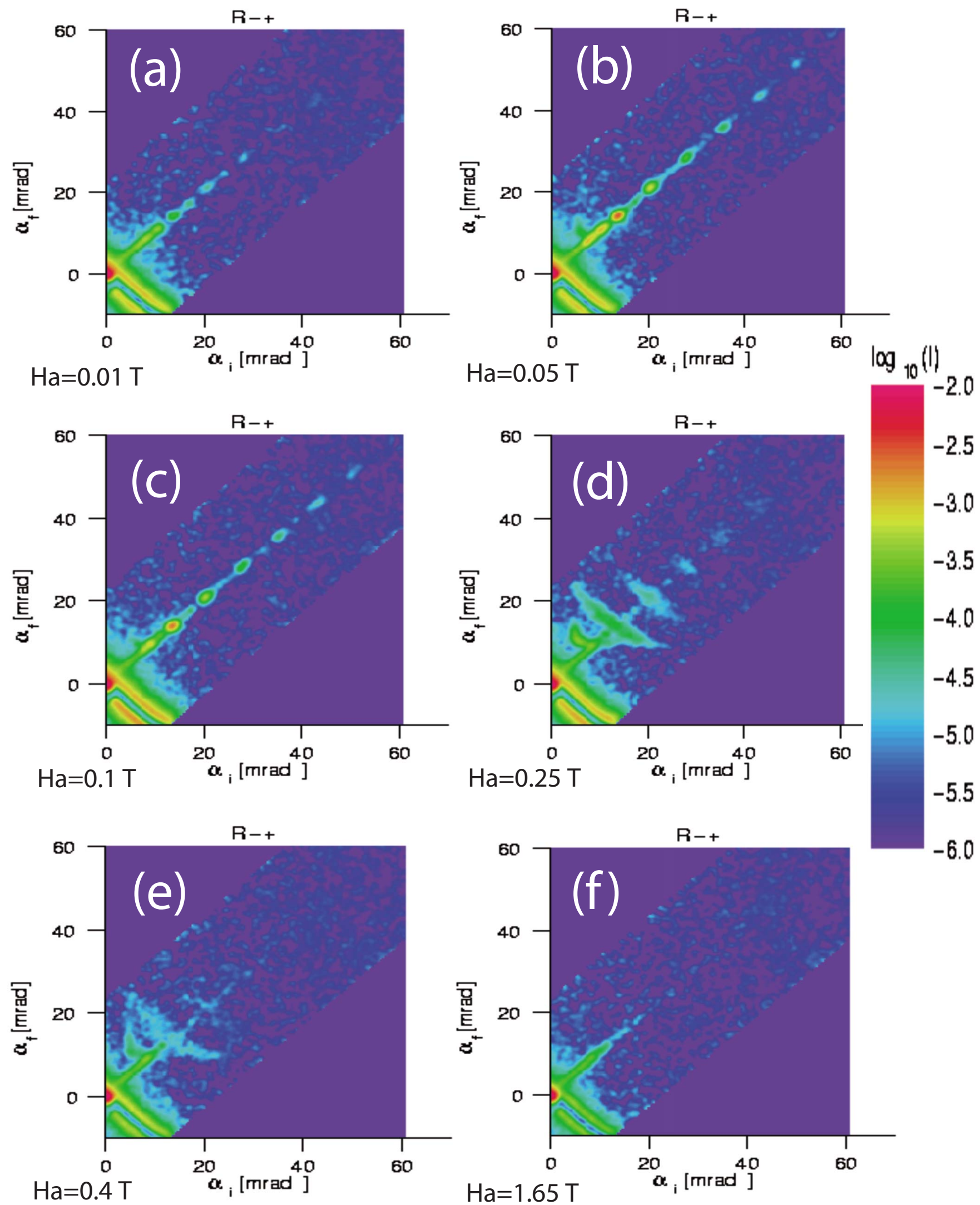

$\mathrm{Ha}=0.4 \mathrm{~T}$

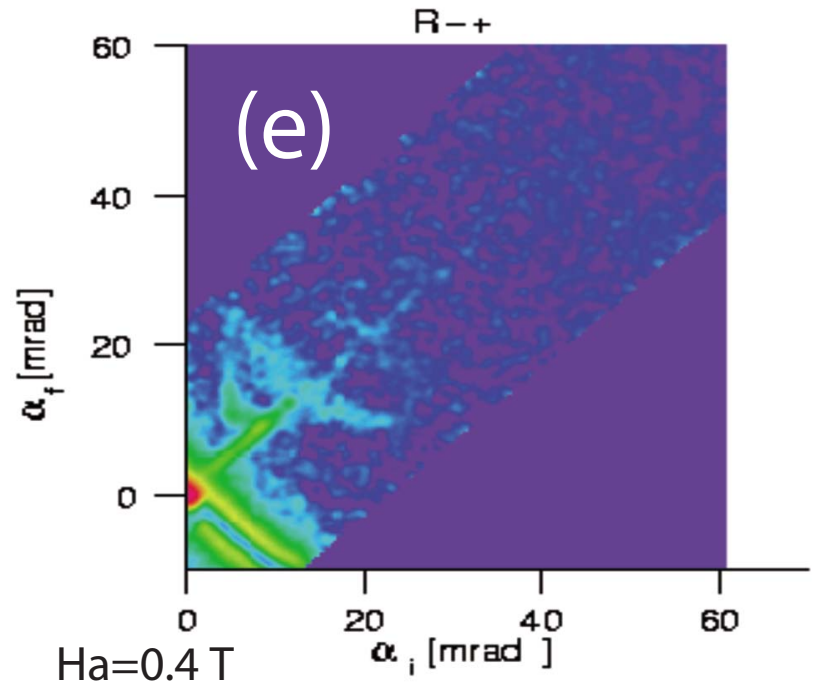

FIG. 6. (Color online) SF intensity maps $\left(R_{-+}\right)$of a Fe/Si ML measured at different $H_{a}$ after negative saturation. The color bar encodes the scattered intensity on a logarithmic scale. 


\section{Neutron scattering signal and the field history}

In a subsequent experiment, we saturated the specimen at $+0.4 \mathrm{~T}$ (instead of $-4.0 \mathrm{~T}$ ) and measured the neutron scattering signal again at $H_{a}=0.25 \mathrm{~T}$, i.e., after positive saturation of the specimen. We find a magnetization state, which is very different from that measured after negative saturation. We compile the corresponding reflectivity patterns along with the SF off-specular intensity map in Fig. 7. Interestingly, no off-specular intensities are visible after positive saturation in clear contrast to the situation after negative saturation (Fig. 6). A disappearance of the off-specular scattering signal is indicative of a larger length scale of the magnetic correlations (larger than $l_{\|}$). Thus, the measurements after negative and positive saturations clearly indicate that the creation of domains or the lateral length scale of magnetic correlations in the twisted state configuration is largely dependent on the field history as well. Moreover, the specular patterns are dissimilar to those observed after negative saturation as the SF reflectivities are more intense.

The history dependence of the domain sizes can stem from the fourfold in-plane anisotropy. Due to symmetry, there are always two energetically equivalent magnetization orientations, if the external field is exactly aligned along an easy or hard axis. On the other hand, if the external field is almost aligned along an easy or hard axis, then the two possible magnetization orientations are slightly different in energy. As a consequence, complicated domain patterns may appear, which will be also dependent on the history. A corresponding discussion of Kerr microcopy images of $\mathrm{Fe}(001)$ single films is given in Ref. 20. The differences in the extent of off-specular intensities for measurements after positive and negative saturation are therefore due to the small differences in the respective magnetic configurations. Such differences can also be related to a small misalignment of the sample axis with respect to the applied field axis. However, as we measure the two cases without altering the experimental setup, we can easily rule out this possibility.

\section{DISCUSSION OF THE RESULTS}

The reflection coefficients can be calculated in the dynamical approach, which is suitable for the low- $Q$ regime, within Parratt's formalism. ${ }^{13}$ In this approach, a model density profile based on prior knowledge of the scattering densities is considered, which should be sufficiently close to the correct one. The scattering lengths and interfacial roughnesses are obtained from the fits in the AF and saturated states, which are confirmed by SQUID and thus have an unambiguous magnetization state. For other, noncollinear states, those values have been kept fixed and only the magnetization angles are fitted. Here, we employ two different models for the magnetization states to fit the neutron scattering data.

(a) The magnetization of each FM layer is uniform and rigid.

(b) The magnetization vector is fanning across the thickness of each FM layer and forms the twisted state. By employing these two models, we explore the alternative possibilities of magnetization configurations at the interface that might have been realized.

\section{A. Simulation within the rigid-state approximation}

\section{Specular scattering}

As a starting point for fitting the specular data, we consider the simplest model, wherein an identical magnetization configuration exists for all of the $\mathrm{Fe} / \mathrm{Si} / \mathrm{Fe}$ trilayers in the ML stack and each Fe layer rotates in a single domain state with the applied field. In other words, this is a rigid-state approximation of the magnetization without any twisting. This configuration essentially minimizes the number of fitting parameters in the routine.

The fits to the specular reflectivities for the NSF and SF channels are carried out by taking into account the nonideal polarization efficiencies of the polarizer and the analyzer. We find reasonable agreement with the nominal values of film thicknesses and the nuclear and magnetic scattering length densities without assuming any interfacial layer between the $\mathrm{Si}$ and Fe. The respective scattering length densities for $b_{\mathrm{Fe}}$ $=7.0910^{-6} \AA^{-2}$ and $b_{\mathrm{Si}}=4.1310^{-6} \AA^{-2}$ considered in the model are close to their nominal values. The details of the fitting procedure are similar to that described earlier. ${ }^{8}$

Figure 8 shows the behavior of $\theta$, i.e., the angle between layer magnetizations $M_{i}$ and $M_{i+1}$, as a function of $H_{a}$, where the layer index ranges from $i=1$ to $i=6$ as the top or bottom Fe layer was found to be decoupled from the rest of the stack. The variation in $\theta$ is found to be monotonic and approaches zero with increasing field strength. A schematic of the scattering geometry is provided in the inset of Fig. 8. In Fig. 9, we plot as filled symbols the symmetric variation in the average magnetization component $\sin \left(\phi_{A}\right)$ with $H_{a}$ in two consecutive layers $i$ and $i+1$ as deduced from the fits to the PNR data.

The fourfold in-plane magnetocrystalline anisotropy of bcc-Fe layers yields a quite symmetric behavior of $\sin \phi_{A}$ for the two coupled layers (Fig. 9), which renders them otherwise practically indistinguishable from each other. However, the SF reflectivities are generally proportional to the mean square of the in-plane magnetization component perpendicular to the polarization direction or the applied field direction $M_{\perp}$, i.e., $\propto\left\langle\sin ^{2}\left(\phi_{A}\right)\right\rangle$, while the NSF reflectivities are proportional to the mean value of the in-plane magnetization component parallel to the polarization axis, i.e., $\propto\left\langle\cos \left(\phi_{A}\right)\right\rangle$. As the sign of the magnetization is encoded in the phase of the SF reflectivities, we can measure only the modulus of $M_{\perp}$

A slight broadening of the Bragg-peaks in the SF reflectivities at $H_{a}=0.25 \mathrm{~T}$ has been observed compared to the nuclear Bragg peaks in the NSF reflectivities. Such a broadening can be attributed to a loss of the vertical correlation through the multilayer stack. However, increased offspecular scattering at the Bragg-peak positions (Fig. 7) indicates the existence of vertical correlation in the stack. Thus, the broadening of the SF AF Bragg peaks is believed to originate from random fluctuations of the coupling strengths across the multilayer. Earlier, we observed a similar broadening of the SF half-order peaks for $\mathrm{AF}$ coupled $\mathrm{Co} / \mathrm{Cu}$ 

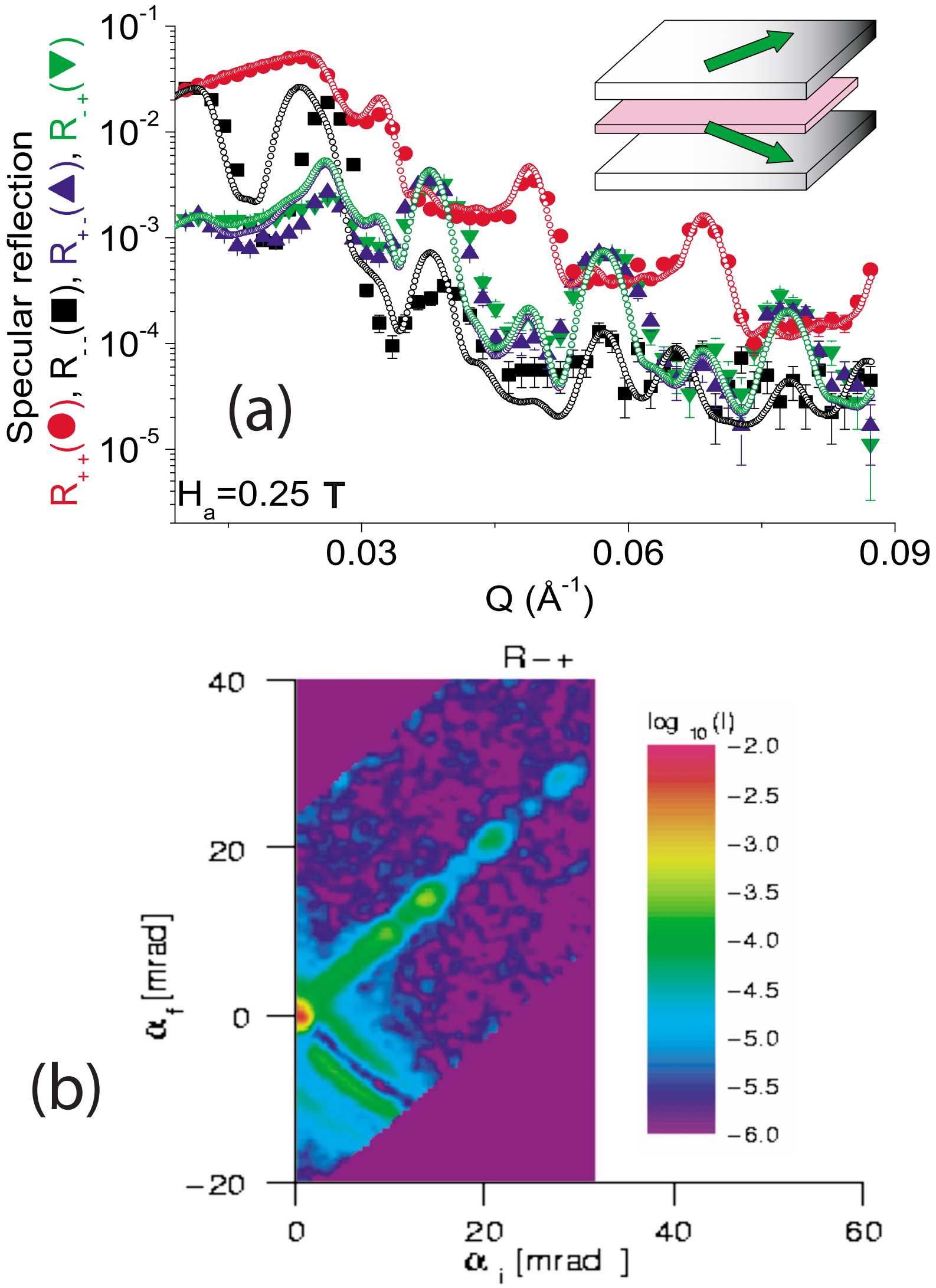

FIG. 7. (Color online) (a) Specular reflectivity patterns for the NSF $\left[R_{++}\right.$(red) and $R_{--}$(black) $]$and SF $\left[R_{-+}\right.$(green) and $R_{+-}$(blue) $]$ channels measured for a Fe/Si ML at $H_{a}=0.25 \mathrm{~T}$ after positive saturation. The solid symbols are data and the open circles are fits to a rigid-state model. (b) SF intensity maps $\left(R_{-+}\right)$at $H_{a}=0.25 \mathrm{~T}$ after positive saturation. The color bar encodes the scattered intensity on a logarithmic scale. 


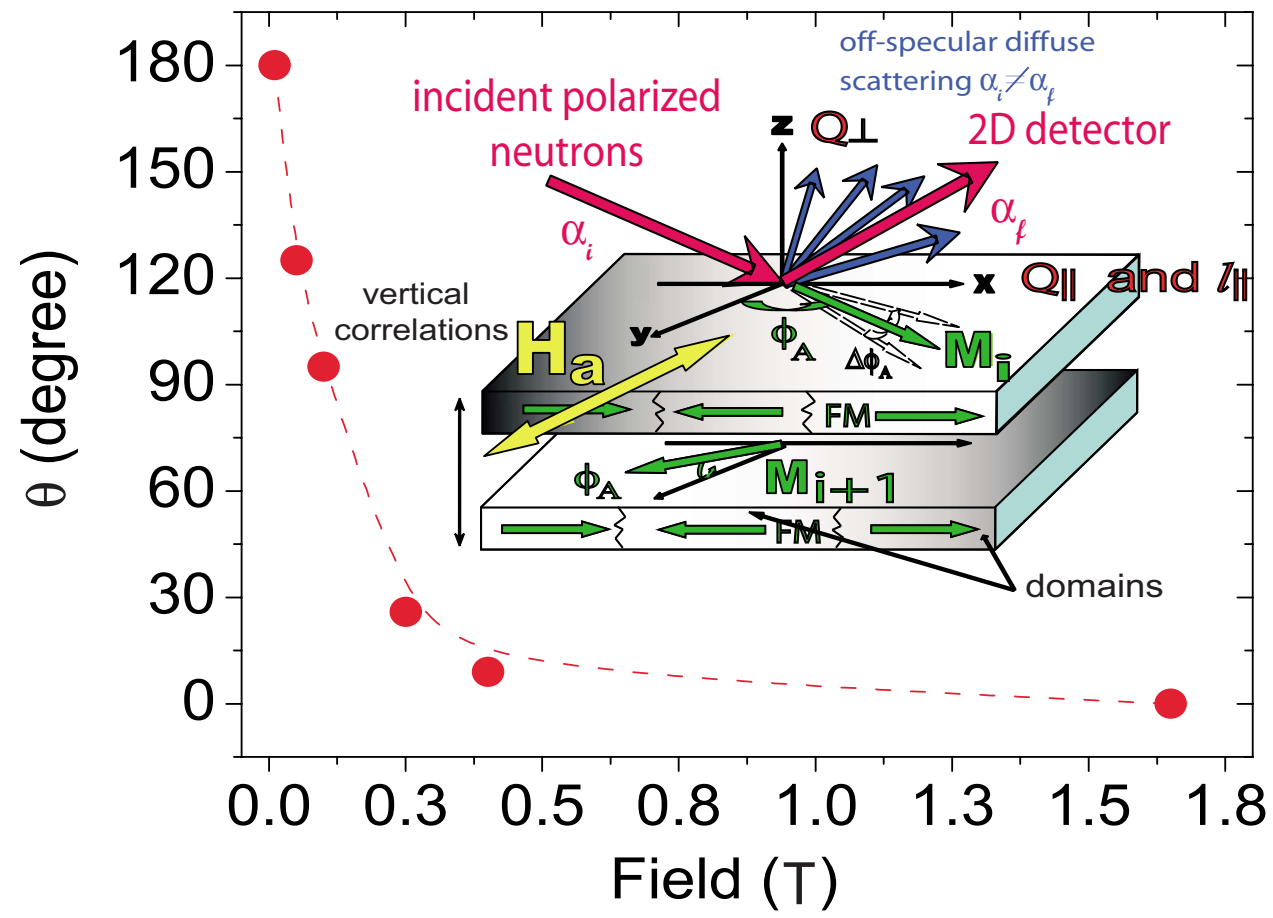

FIG. 8. (Color online) Variation in the angle $\theta$ between $M_{i}$ and $M_{i+1}$ as a function of the applied field. The error bars $\left( \pm 3^{\circ}\right)$ are indicated by the symbol size. The dashed line is a guide to the eye. The angles are extracted from the fits of the specular data to the rigid-state model. Inset: schematic of the scattering geometry.

multilayers. ${ }^{14}$ For simplicity, we do not take into account such fluctuations in our models. A small deviation $\phi_{A}$ in the layer magnetizations from the applied field direction does not make any significant difference in the magnitude of the parallel component (NSF intensities): $M_{\|} \propto\left\langle\cos \left(\phi_{A}\right)\right\rangle$ $\approx\left\langle 1-\left(\phi_{A}\right)^{2} / 2\right\rangle$, whereas SF scattering is sensitive to $M_{\perp}$ $\propto\left\langle\sin ^{2}\left(\phi_{A}\right)\right\rangle \approx \phi_{A}^{2}$. Thus, for small $\phi_{A}$, the difference between the NSF specular signals becomes insignificant. This explains the differences between the widths of the peaks for the NSF and SF channels.
The overall quality of the least square fits for all other applied fields is of a reasonably high quality, confirming the apparent validity of the rigid-state approximation model except for the intermediate field range around $H_{a}=0.25 \mathrm{~T}$. Here, even though the NSF reflectivities reasonably agree well with the simulated patterns, the SF intensities are clearly in discrepancy. Around $H_{a}=0.25 \mathrm{~T}$ and above, the intensities in the SF channel are mostly within the NSF background. The NSF background arises from the inefficiencies of the polarizer and analyzer components of the reflectometer

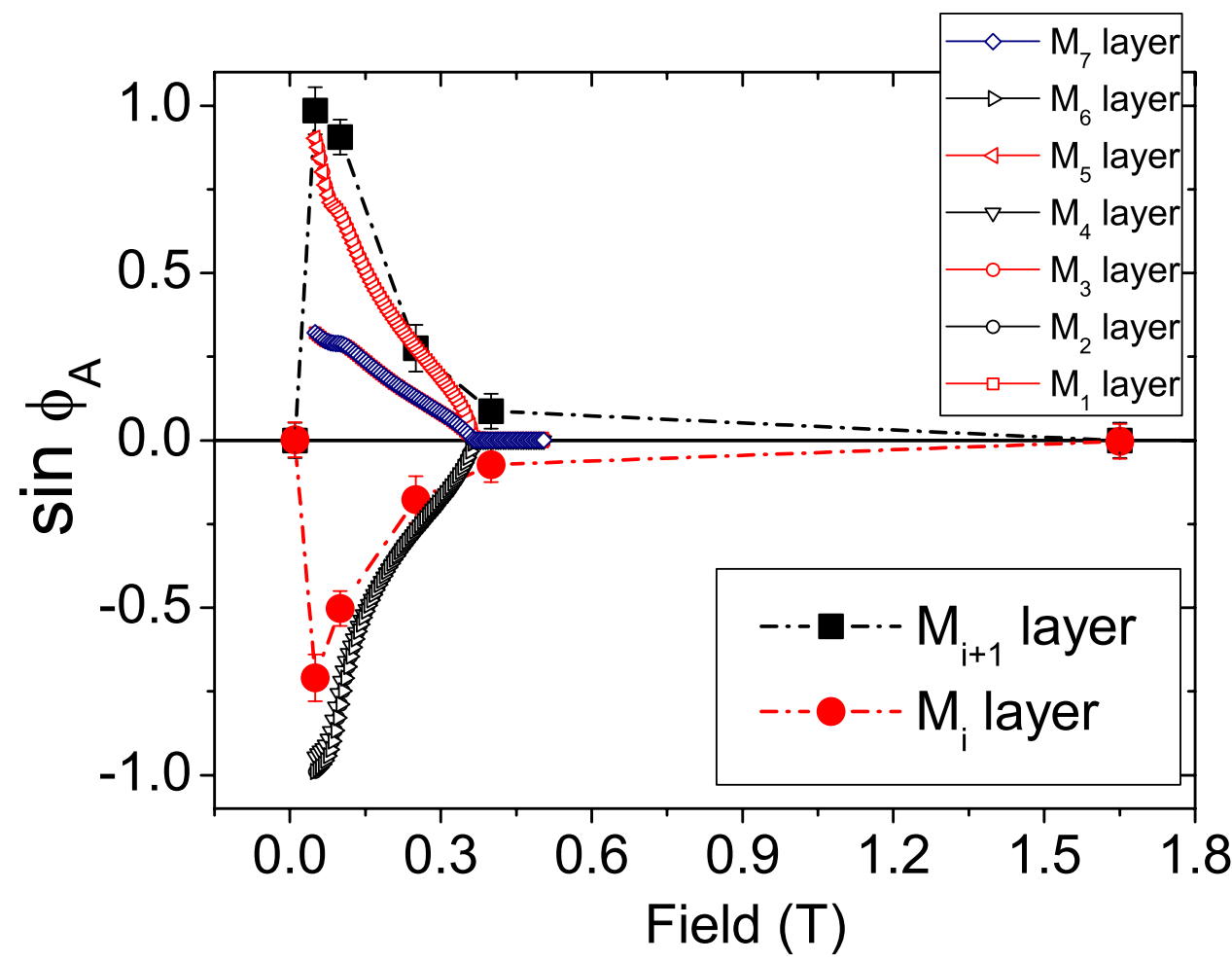

FIG. 9. (Color online) Field dependence of $\sin \left(\phi_{A}\right)$ for $M_{i}$ (filled red dots) and $M_{i+1}$ (filled black squares) as extracted from fitting the specular data to the rigid-state model. The directions of all Fe layers in the ML as deduced from the micromagnetic simulation of the SQUID curve are plotted for comparison by using the open symbols. 
(typical inefficiencies are $\approx 6 \%$, i.e., we can obtain $\approx 94 \%$ of polarization for our specular reflection, and in our model, we have incorporated this deviation). Such inefficiencies can stem from nonideal supermirrors (polarizer and analyzer) and the stray fields of the electromagnet (conflicting with the compensation field of the flippers) that is used to provide the field to the sample. This is evident when we compare the SF intensities observed at all other field values to those measured at magnetic saturation $\left(H_{a}=1.65 \mathrm{~T}\right)$, where the magnetizations are completely aligned with the field and we do not expect any residual SF signal.

At the field value of $H_{a}=0.25 \mathrm{~T}$, the deviation of the theoretical specular reflectivity pattern from the experimental one is mainly caused by (i) an overestimation of the SF specular reflectivity (a simple consideration of a decrease in the $M_{\perp}$ component would render the corresponding $M_{\|}$components far more in disagreement with the model) and (ii) our approximation of a rigid-state model instead of assuming a more complex twisted magnetization configuration. The off-specular intensities are distinctly prominent in the case of the measurements at $H_{a}=0.25 \mathrm{~T}$ (Fig. 6). This enhancement indicates an instability in the system and can be accounted for within the DWBA by considering fluctuations of the magnetizations around their mean direction. A change in slope of $\theta$ versus $H_{a}$ (which looks like a hyperbola) around $H_{a}=0.25 \mathrm{~T}$ in Fig. 8 signifies the instability at this particular field value. Thus, we believe that a proper estimation of $\theta$ in the region around the critical field value is practically impossible, at least within the rigid-state approximation model.

In the BLS experiments in Ref. 5, the twisted state configuration was observed over a large field range, whereas the neutron data indicate the twisted state in a narrow fields range around $H_{a}=0.25 \mathrm{~T}$ only. This difference between BLS and PNR comes about because PNR can only distinguish the twisted state from the rigid configuration when the deviation of magnetization in each layer from the mean orientation is large enough. This is the case for $H_{a}=0.25 \mathrm{~T}$, but at other fields, this deviation is too small to be resolved by PNR. Additionally, the situation is even more difficult in the case of a ML compared to the trilayer system investigated by BLS.

The fits to the reflectivity data measured after positive saturation (Fig. 7) are carried out in a similar manner, as described above. The angles, which the layer magnetizations $\left(M_{i}\right.$ and $\left.M_{i+1}\right)$ subtends with the field axis $\left(\phi_{A}\right)$, are found to deviate stronger from the field direction compared to that obtained at the same field value but after negative saturation. For the starting situation after positive saturation, the angle between the magnetization directions of the two consecutive layers in the stack $(\theta)$ is estimated to be $47^{\circ} \pm 3^{\circ}$ instead of $\approx 26^{\circ}$ after negative saturation. This difference obviously accounts for the larger SF specular intensities. In this context, it is notable that the SQUID data exactly shows the same longitudinal magnetization for positive and negative sweep directions. Therefore, the history dependent difference must be purely attributed to the different lateral domain sizes and configurations.

Interestingly, the fits to the reflectivity patterns in Fig. 7 are in better agreement with the data (even within the simple rigid-state approximation) compared to that found at the same field value in the measurement after negative saturation. This is mainly because the SF signal is truly specular (distinctly above the NSF contamination) as no off-specular intensities are visible above the instrumental background [see Fig. 7(b)]. In the case of the measurement after negative saturation at $H_{a}=0.25 \mathrm{~T}$ (Fig. 5), the SF signal is mostly due to the off-specular scattering. However, the agreement of the model with the experimental data (even after positive saturation) is still far from being convincing. We therefore conclude that the magnetization state at this field value cannot be simply described by means of a rigid-state model irrespective of the field history. We rather have to invoke a more realistic model based on a twisted magnetization configuration.

It is worthwhile to mention that without the accessibility to measure the off-specular spectra, it would not have been possible to distinctly identify a twisted state. This is because, as when the domain sizes are relatively small, the extraction of the true specular intensities above the diffuse background is rather ambiguous, whereas, for large domain sizes, as the diffuse intensities are absent, the extraction is much more reliable. Thus, the configuration (realized after positive saturation) that possesses larger domains (larger than $l_{\|}$) and remains twisted is a necessity in this investigation by PNR.

\section{Off-specular scattering}

In an effort to simulate the off-specular scattering results within the DWBA — still assuming a rigid-state model-we consider the magnetization to randomly fluctuate from domain to domain around the mean angle $\phi_{A}$. The perturbation includes an antiferromagnetic character as the intensities are located at the half-order Bragg sheets. Furthermore, the antiferromagnetic domains are extended throughout the film thickness. This conclusion can be drawn from the widths of the off-specular Bragg sheets. The off-specular and specular SF and NSF scattering signals are calculated within the supermatrix formalism ${ }^{21}$ and are found to reproduce almost all of the details, such as the asymmetry in the low- $Q$ Bragg sheet, in the intensity distributions.

Figure 10 shows the simulated SF intensity maps (within the DWBA approach) for the different configurations of the magnetization state corresponding to the measured data at $H_{a}=0.25 \mathrm{~T}$ after negative [Figs. 10(a) and 10(b)] and positive [Figs. 10(c) and 10(d)] saturation, respectively. We have considered different magnetic correlation lengths $(\xi)$ for the two cases. A schematic of the magnetic domain configuration is shown in Fig. 10(e). It is found that the simulation considering $\xi=0.5 \mu \mathrm{m}$ and a fluctuation of the average magnetization around their mean direction $(\Delta \phi)$ is able to capture the magnetization state realized after negative saturation. The full width half maximum of such a fluctuation is around $30^{\circ} \pm 5^{\circ}$. In the case of the magnetic configuration after positive saturation, a simulation with $\xi=0.5 \mu \mathrm{m}$, which is close to the probing limit of our instrument and $\Delta \phi=0^{\circ}$, yields a result that reasonably well compares to the experimental data. The fluctuations $(\Delta \phi)$ of the magnetization from domain to domain would obviously be irrelevant for a singledomain configuration. Thus, given the limit of the instrument in resolving lateral structures, we believe that the situation 
after negative saturation
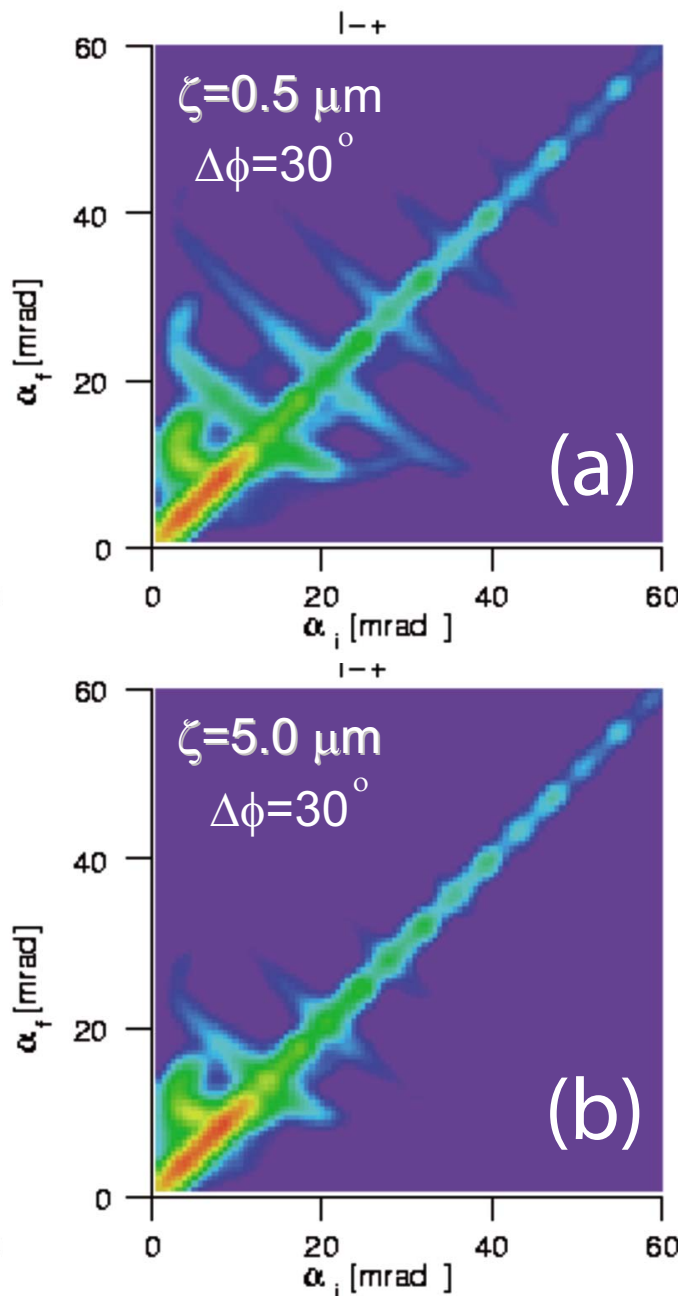

after positive saturation

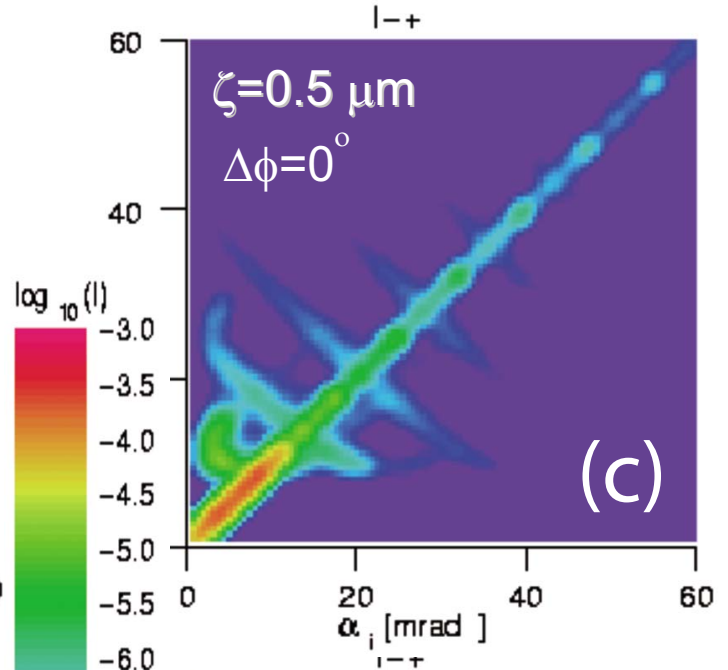

$-6.5$

$-7.0$

$-7.5$

$-8.0$

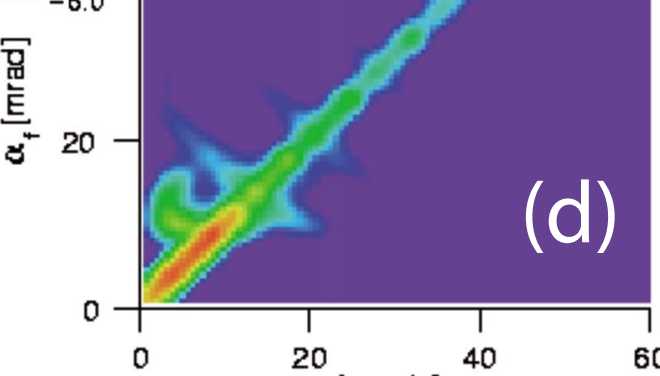

(e)

$\alpha_{i}[\operatorname{mrad}]$

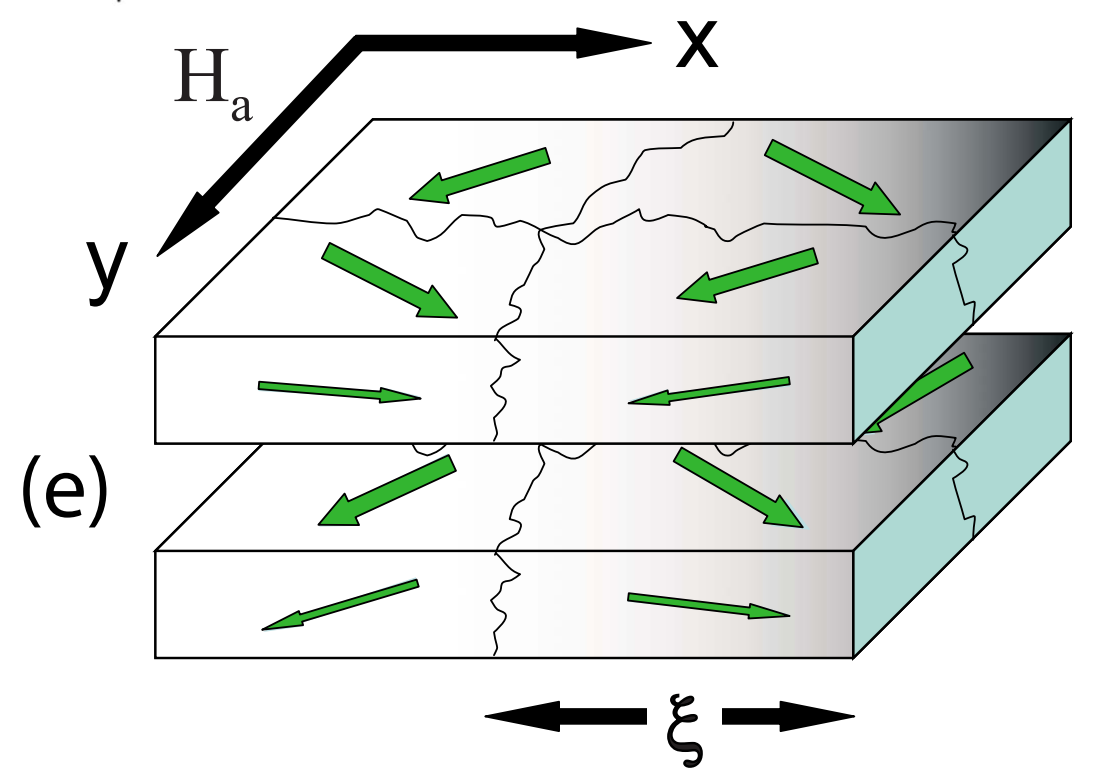

FIG. 10. (Color online) Simulated SF intensity maps $\left(R_{-+}\right)$of a Fe/Si ML for two different magnetic correlation lengths $\xi=0.5$ and $5.0 \mu \mathrm{m}$ and two different degrees of fluctuations $\Delta \phi=0^{\circ}$ and $30^{\circ}$. By comparing to the data measured at $H_{a}=0.25 \mathrm{~T}$, (a) and (b) correspond to the magnetization state after negative and (c) and (d) correspond to the magnetization state after positive saturation, respectively. The color bar encodes the scattered intensity on a logarithmic scale. (e) Schematic of the magnetic domain structure considered in the DWBA modeling. 


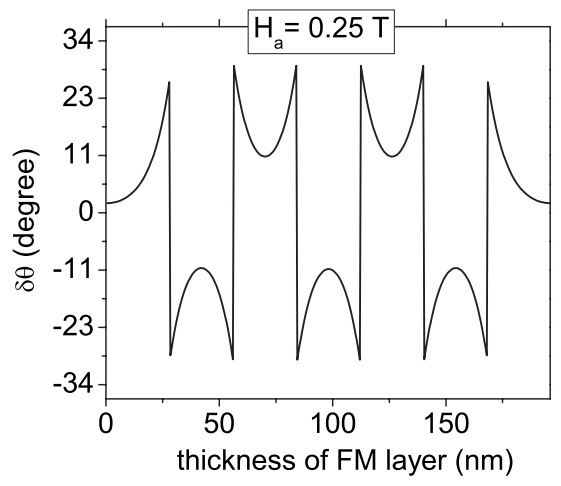

FIG. 11. Angular deviation of the magnetization from the applied field direction $(\delta \theta)$ derived from fitting the twisted state model to the SQUID data at $H_{a}=0.25 \mathrm{~T}$. The $x$ axis spans the total FM thickness of all of the seven Fe layers but neglects the thickness of the Si interlayers.

observed after positive magnetic saturation is that of a single-domain state, whereas it is a multidomain state after negative saturation.

\section{B. Simulation within the twisted state model}

\section{Micromagnetic simulation}

In Sec. IV A, we have seen the failure of the uniform magnetization model in fitting the data of our experiments at $H_{a}=0.25 \mathrm{~T}$ for a situation, in which the sample was previously saturated at $\pm 4.0 \mathrm{~T}$. In an effort to improve our understanding of these results, we introduce the twisted state model, which was inferred earlier from the BLS experimental results on similar systems. ${ }^{5}$ The spatial variation of the magnetization vector was extracted from micromagnetic simulations, which allow vertical variations only. The orientation of Fe moments relative to the applied field was obtained by minimizing the energy equation. We take into account the identical coupling parameters for all of the interlayers; otherwise. we would get too many fit parameters. The magnetic configuration thus deduced was then used to analyze the neutron reflectivity data within the dynamical format. The spin configuration within the FM layers in the system extracted from the fits to the SQUID curve is shown in Fig. 11. The deviation from the applied field direction gradually increases from the middle of the layer toward the interfaces, where the $\mathrm{AF}$ coupling acts strongest. A schematic illustrating such a chiral spin arrangement is given in Fig. 12.

\section{Specular scattering in the twisted stated model}

Figure 13 shows the simulations of the reflectivity pattern measured after positive saturation at $H_{a}=0.25 \mathrm{~T}$. Each set of simulated patterns [Figs. 13(a)-13(c)] corresponds to the different combinations of $J_{1}$ and $J_{2}$ values obtained from the micromagnetic fits to the SQUID data. Each Fe layer is now divided into 28 sublayers with a thickness of $1.0 \mathrm{~nm}$ for each sublayer. The magnetization directions follow the scheme shown in Fig. 11. From the three sets of $J_{1}$ and $J_{2}$, we obtain three sets of magnetization angles for each of the sublayers. We particularly choose to simulate the reflectivity patterns

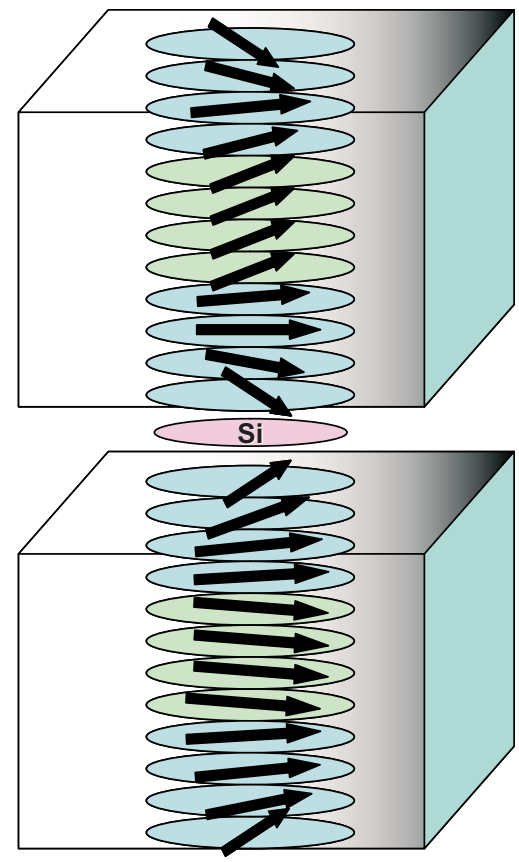

FIG. 12. (Color online) Schematic of the magnetization profile of two adjacent $\mathrm{Fe}$ layers corresponding to the experimental data obtained at $H_{a}=0.25 \mathrm{~T}$.

for the state realized after positive saturation because in the absence of SF off-specular scattering, the extraction of the specular data is more reliable.

The three sets of $J_{1}, J_{2}$ values considered here are indicated in Fig. 13. The simulations in Fig. 13(a) correspond to the situation wherein the coupling constants are reduced by $20 \%$ from the values, which have been obtained from the fits to the SQUID curve at the critical field. Figure 13(b), however, shows the best agreement of the experimental with the simulated patterns, which is obtained by reducing $J_{1}$ and $J_{2}$ by $10 \%$ compared to the SQUID results. Fits with the $J_{1}, J_{2}$ values from SQUID are finally shown in Fig. 13(c). The changes in the fitted reflectivity curves appearing after a $10 \%-20 \%$ reduction of $J_{1}$ and $J_{2}$ are indicative of the sensitivity of our estimation of the magnetization state by PNR.

We now focus the fits to the data shown in Fig. 7 for the rigid configuration and those in Fig. 13(b) for the twisted state model. One can clearly see that the quality of the fit is improved if we consider the twisted state model. Thus, following an energy minimization, micromagnetic simulations can provide a physically realistic magnetic configuration of the Fe moments that can-in turn-lead to better agreement of the simulated patterns with the observed neutron reflectivity patterns. However, the corresponding coupling constants $\left(J_{1}=-2.0 \mathrm{~mJ} / \mathrm{m}^{2}, J_{2}=-0.27 \mathrm{~mJ} / \mathrm{m}^{2}\right)$ yielding the best fit in Fig. 13(b) are lower than those determined from the SQUID curve, although they best represent the neutron reflectivity patterns. Such small differences in $J_{1}, J_{2}$ from SQUID and PNR can be attributed to the fact that SQUID measures the net $M_{\|}$component of the sample, whereas PNR measures the Fourier transform of the electron spatial distribution (along the thickness) probed by the elastic magnetic scattering of the neutrons averaged over its coherence length 


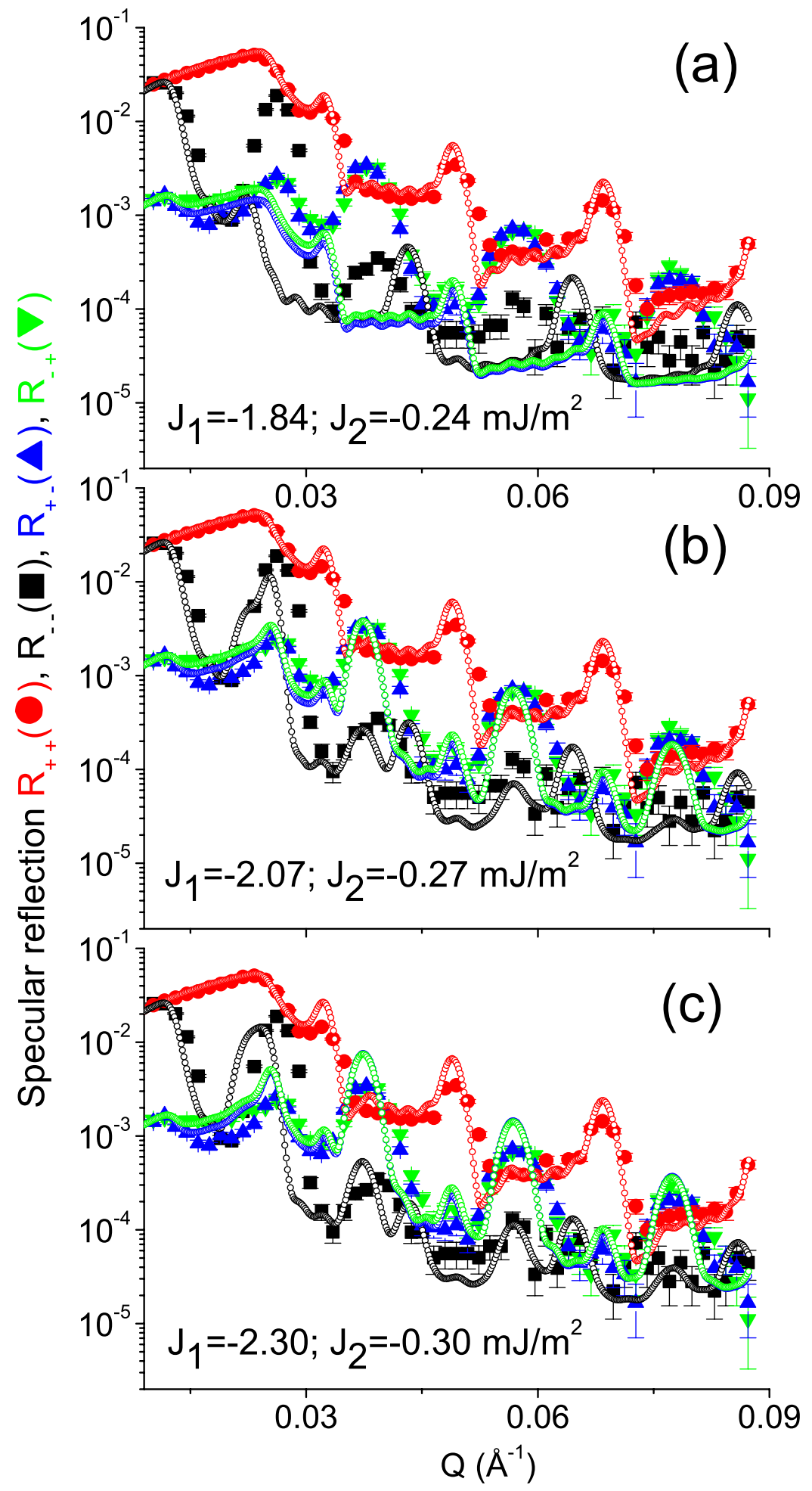

FIG. 13. (Color online) Specular reflectivity patterns for the NSF $\left[R_{++}\right.$(red) and $R_{--}$(black) $]$ and $\mathrm{SF}\left[R_{-+}\right.$(green) and $R_{+-}$ (blue)] channels for a $\mathrm{Fe} / \mathrm{Si} \mathrm{ML}$ measured at $H_{a}=0.25 \mathrm{~T}$ after positive saturation. The solid symbols are data and the open circles are fits to the twisted state model carried out with different combinations of $J_{1}$ and $J_{2}$ parameters as indicated.

in the sample plane. Thus, information that can be extracted for a multilayer stack largely depends on the range of the $Q$ vectors that can be probed (limited by the background intensity) with suitable resolution and on the model that is invoked to fit the reflectivity profiles. We emphasize that it would not have been possible to achieve such agreement of the theoretical and the experimental reflectivities without the support of the micromagnetic simulations.
In this regard, one must not forget that the PNR data extraction procedure and its interpretation is largely dependent on the relative size of the domains and $l_{\|}$of the neutron beam. The analysis becomes easier when the size of the domains are either larger or smaller than $l_{\|}$. For larger domains, the signal is a sum of incoherent average over several coherent volumes, whereas for smaller domains, the signal is a mean of the average magnetization (the scale of averaging 
being greater than that of $l_{\|}$). The situation becomes rather complicated when the two length scales are comparable to each other and this is what has been encountered in the present case. Here, the domain sizes are found to be $\approx 0.5 \mu \mathrm{m}$, i.e., smaller than $l_{\|}$after negative saturation, and $\approx 5.0 \mu \mathrm{m}$, i.e., about equal $l_{\|}$to after positive saturation. As the domain sizes tends to approach the size of $l_{\|}$, the line shape of the reflected beam substantially becomes distorted. ${ }^{22}$ The superimposition of two comparable potentials, i.e., the mean optical potential (giving rise to specular scattering) and the fluctuations around the mean potential (responsible for off-specular scattering), makes it difficult to unambiguously quantify the PNR data. Within the DWBA, the reference Hamiltonian must be exactly solved and the Hamiltonian due to fluctuations are to be treated as a perturbation to the reference one. For comparable potentials, the DWBA may no longer be applicable as the fluctuation cannot be accounted for as a perturbation and this therefore becomes an inherent problem.

Moreover, the range of $\delta \theta\left( \pm 10^{\circ}\right)$ is rather small. The standard deviation of the rotating moments from the average uniform magnetization within each layer renders very small changes in $\phi_{A}$. The situation becomes even more pronounced for a stack of multiple layers. The differences in the $M_{\|}$components of the rotating moments within each layer are rather small, which may become difficult to be resolved by PNR. This limitation may have compromised a more refined estimation of the twisting chirality in the present case. PNR generally suffers from a low signal-to-noise ratio at higher $Q$ values. However, with the advent of brighter sources in the future, such an investigation will possibly become feasible even in the case of trilayers $(\mathrm{Fe} / \mathrm{Si} / \mathrm{Fe})$.

\section{SUMMARY AND CONCLUSION}

The magnetization profile of strongly AF-coupled $\mathrm{Fe} / \mathrm{Si}$ multilayers have been probed by depth sensitive and vector magnetometric polarized neutron scattering. We have combined micromagnetic simulations with our neutron scattering data in order to explore the magnetization depth profile across the ML stack. The aim of this investigation is to examine the twisted state of the magnetization in buried layers by using a complementary method, i.e., probing the Fourier transform of the magnetization profile with PNR rather than indirectly inferring the state by analyzing the depth-limited spin-wave frequencies in BLS.

The magnetization angles for the layers in the stack have been extracted from the specular reflectivity patterns as a function of the applied field. The neutron scattering data have been analyzed by considering two different models,

(a) one in which the $\mathrm{Fe}$ moments remain rigid (do not rotate) within each FM layer; and

(b) the other when they are in a twisted state (progressively rotate across the thickness of each FM layer).

In our investigation, the magnetization profile has been found to significantly deviate from the simplistic rigid-state model to a twisted ground state of the magnetization. This is particularly pronounced around an intermediate (critical) applied field. Micromagnetic simulations, which are in agreement with the SQUID data, provide a physically realistic energy-minimized configuration for the rotating moments and has been proved to be essential for the neutron data analysis.

Interestingly, at the critical field, we observe enhanced off-specular scattering in the SF channels located at the AF Bragg-peak positions. These off-specular scattering contributions are attributed to the presence of the associated buried magnetic domains. Moreover, the length scale of these domains is observed to be larger or smaller than $l_{\|}$, depending on the field history the specimen has undergone. The estimation of the chirality in the spin configuration can be difficult in the absence of $M_{\perp}$ components (in the SF channel) due to the formation of domains smaller than $l_{\|}$or when the two length scales are comparable to each other.

\section{ACKNOWLEDGMENTS}

The authors wish to thank R. Schreiber for helping in preparing the sample, Thomas Brückel for insightful discussions, and P. Grünberg for his interest.

\footnotetext{
*Present address: Hahn-Meitner-Institut Berlin GmbH, Glienicker Strasse 100, D-14109 Berlin, Germany. Author to whom correspondence should be addressed; amitesh.paul@hmi.de

†Present address: Institut für Angewandte Physik, Corrensstrasse 2-4, Westfälische Wilhelms-Universität, D-48149 Münster, Germany.

${ }^{1}$ P. Grünberg, R. Schreiber, Y. Pang, M. B. Brodsky, and H. Sowers, Phys. Rev. Lett. 57, 2442 (1986).

${ }^{2}$ M. N. Baibich, J. M. Broto, A. Fert, F. Nguyen Van Dau, F. Petroff, P. Etienne, G. Creuzet, A. Friederich, and J. Chazelas, Phys. Rev. Lett. 61, 2472 (1988).

${ }^{3}$ G. Binasch, P. Grünberg, F. Saurenbach, and W. Zinn, Phys. Rev. B 39, 4828 (1989).

${ }^{4}$ R. R. Gareev, D. E. Bürgler, M. Buchmeier, R. Schreiber, and P. Grünberg, J. Magn. Magn. Mater. 240, 237 (2002).
}

${ }^{5}$ M. Buchmeier, B. K. Kuanr, R. R. Gareev, D. E. Bürgler, and P. Grünberg, Phys. Rev. B 67, 184404 (2003).

${ }^{6} \mathrm{Ph}$. Bauer, M. Sajieddine, C. Dufauer, K. Cherifi, G. Marchal, and Ph. Mangin, Europhys. Lett. 16, 307 (1991).

${ }^{7}$ D. Haskel, Y. Choi, D. R. Lee, J. C. Lang, G. Srajer, J. S. Jiang, and S. D. Bader, J. Appl. Phys. 93, 6507 (2003).

${ }^{8}$ A. Paul, E. Kentzinger, U. Rücker, D. E. Bürgler, and P. Grünberg, Phys. Rev. B 70, 224410 (2004).

${ }^{9}$ C. Dufour, K. Cherifi, G. Marchal, Ph. Mangin, and M. Hennion, Phys. Rev. B 47, 14572 (1993).

${ }^{10}$ K. V. O’Donovan, J. A. Borchers, C. F. Majkrzak, O. Hellwig, and E. E. Fullerton, Phys. Rev. Lett. 88, 067201 (2002).

${ }^{11}$ M. R. Fitzsimmons, S. Park, K. Dumesnil, C. Dufour, R. Pynn, J. A. Borchers, J. J. Rhyne, and Ph. Mangin, Phys. Rev. B 73, 134413 (2006). 
${ }^{12}$ B. P. Toperverg, Polarized Neutron Scattering (Forschungszentrum Jülich, Germany, 2002), Vol. 12, p. 247.

${ }^{13}$ J. F. Anker and G. P. Felcher, J. Magn. Magn. Mater. 200, 698 (1999).

${ }^{14}$ A. Paul, E. Kentzinger, U. Rücker, D. E. Bürgler, and P. Grünberg, Eur. Phys. J. B 45, 249 (2005); A. Paul, E. Kentzinger, U. Rücker, D. E. Bürgler, and Th. Brückel, Phys. Rev. B 73, 094441 (2006).

${ }^{15}$ M. Buchmeier, R. Schreiber, D. E. Bürgler, and C. M. Schneider, arXiv:0803.1599 (unpublished).

${ }^{16}$ M. J. Donahue and D. G. Porter, “OOMMF User's Guide, Version 1.0," National Institute of Standards and Technology Interagency Report No. NISTIR 6376, 1999 (unpublished).
${ }^{17}$ A. Paul, M. Buchmeier, C. Schneider, and Th. Brückel, J. Appl. Phys. 101, 123913 (2007).

${ }^{18}$ G. S. Krinchik and V. A. Artemev, Sov. Phys. JETP 26, 1080 (1968).

${ }^{19}$ Handbook of Chemistry and Physics, 82nd ed., edited by D. R. Lide (CRC, Boca Raton, FL, 2002).

${ }^{20}$ S. Yan, R. Schreiber, P. Grünberg, and R. Schäfer, J. Magn. Magn. Mater. 210, 309 (2000).

${ }^{21}$ B. P. Toperverg, A. Rühm, W. Donner, and H. Dosch, Physica B (Amsterdam) 267, 198 (1999).

${ }^{22}$ B. P. Toperverg, V. V. Lauter-Pasyuk, H. J. Lauter, and A. Vorobiev, Physica B (Amsterdam) 356, 51 (2005). 